\title{
Angiolipomatosis renal y linfangioleiomiomatosis, su relación con la enfermedad de Bourneville
}

\author{
Ponce Díaz-Reixa J, Barbagelata López A, Romero Selas E, Marcos Rodríguez P*, \\ Sánchez Rodríguez-Losada J, Álvarez Castelo L, Fernández Rosado E, González Martín M.
}

Servicio de Urología y *Neumología. Hospital Juan Canalejo. A Coruña.

Actas Urol Esp 2006: 30 (4): 386-393

\section{RESUMEN \\ ANGIOLIPOMATOSIS RENAL Y LINFANGIOLEIOMIOMATOSIS, SU RELACIÓN CON LA ENFERMEDAD DE BOURNEVILLE}

Introducción: Los angiomiolipomas renales son poco frecuentes en la actividad urológica. La linfangioleiomiomatosis es una rara enfermedad pulmonar que afecta mayoritariamente a mujeres en edad reproductiva. La Esclerosis Tuberosa afecta a diversos órganos, incluyéndose entre sus presentaciones, estas dos entidades.

Material y método: Se revisan de forma retrospectiva los pacientes atendidos en nuestro centro, haciendo referencia a tres casos representativos de la enfermedad. Se expone su manejo clínico y Resultados: Asimismo se realiza una revisión de la literatura indexada, que nos permita establecer un protocolo de actuación.

Resultados: Entre 1990 y 2004, han sido atendidos 8 pacientes con angiomiolipoma renal. 5 mujeres (62\%) y 3 hombres (38\%). La media de edad es de 52,3 años (29-69). En 3 pacientes (todas mujeres) se diagnosticó también linfangioleiomiomatosis.

Conclusiones: La enfermedad de Bourneville es poco frecuente. Los angiomiolipomas que se asocian, suelen ser múltiples, bilaterales, tendentes al crecimiento y requieren más intervenciones. El diagnóstico precoz permite prevenir futuras complicaciones mediante vigilancia, embolización arterial selectiva o cirugía conservadora. Es importante el tamaño y la presencia de sintomas para decidir la mejor terapia. Las pacientes jóvenes con linfangioleiomiomatosis pulmonar deben ser advertidas del uso de preparados estrogénicos y evitar embarazos.

Palabras clave: Angiomiolipoma Renal. Linfangioleiomiomatosis pulmonar. Enfermedad de Bourneville. Síndrome Tuberosis Esclerosa.

\section{ABSTRACT \\ RENAL ANGIOMYOLIPOMATOSIS AND PULMONARY LYMPHANGIOMYOMATOSIS. IT’S RELATIONSHIP WITH BOURNEVILLE SYNDROME}

Introduction: Renal angiomyolipomas are not very frequent in urologic activities. Pulmonary lymphangioleiomyomatosis is a rare disease that occurs mainly in women of reproductive age. Tuberous sclerosis shows lesions to different organs, including these both entities.

Material and method: We retrospectively analyze patients treated in our center. Three most representatives cases are reported. An indexed literature review is done, in order to establish a clinical protocol to manage these kinds of patients.

Resultados: Between 1990 and 2004, eight patients have been attended with an angiomyolipoma renal diagnostic. Five women (62\%) and three men (38\%). Medium age is 52,3y (29-69). In 3 of them (all women), it was also done a diagnostic of pulmonary lymphangioleiomyomatosis.

Conclusions: Bourneville syndrome is not very frequent. Associated angiomyolipomas usually are multiple, bilateral, with tender to grow and require more interventions. Previous diagnostic let us prevent future complications with vigilance, selective arterial embolization and nephron-spare surgery. Tumour size and the presence of symptoms are more decisive to decide best therapy alternative. Young patients with pulmonary lymphangioleiomyomatosis should be advise against pregnancy and the use of preparations containing estrogens. 
L os angiomiolipomas renales (AML) son tumores benignos o hamartomas de origen mesenquimal, compuestos por vasos sanguíneos, células musculares lisas y tejido adiposo maduro. Generalmente son asintomáticos y cuando presentan sintomas, los más frecuentes son el dolor en flanco, hemorragia retroperitoneal, hematuria o sintomas de pielonefritis. La TAC abdominal los describe como masas bien definidas de tejido blando, de contenido densidad grasa y que pueden medir entre escasos centimetros hasta mayores de $10 \mathrm{~cm}$. La biopsia renal no es necesaria.

La linfangioleiomiomatosis (LAM) es una rara entidad, que afecta mayoritariamente a los pulmones, pero también puede afectar a riñones, sistema linfático, hígado, útero y páncreas. Predominantemente se presenta en mujeres, durante la edad gestacional. Los síntomas de presentación más frecuentes son disnea paroxística, tos irritativa crónica y neumotórax. Las manifestaciones extrapulmonares incluyen masas en ganglios linfáticos abdominales y pélvicos, ascitis quilosa y angiolipomas renales. La TAC Torácica de Alta Resolución muestra quistes parenquimatosos de pared fina, de diámetro entre pequeños milímetros y varios centímetros. El diagnostico definitivo se confirma mediante biopsia pulmonar.

La esclerosis tuberosa, complejo tuberosis esclerosa (TSC) o Enfermedad de Bourneville, es una enfermedad genética y multisistémica de incidencia baja. Provoca tumores benignos de crecimiento en cerebro y otros órganos vitales como riñón, pulmón, corazón; también en ojos y piel. Comúnmente afecta al sistema nervioso central; Aparecen 6.000 casos anuales en el mundo con igual distribución de género y étnica.

\section{MATERIAL Y MÉTODO}

En nuestra serie, entre 1990 y 2004, han sido atendidos 8 pacientes con el diagnóstico de angiomiolipoma renal. 5 mujeres (62\%) y 3 hombres (38\%). La media de edad es de 52,3 años (29-69). En 3 pacientes (todas mujeres) se diagnosticó también linfangioleiomiomatosis; una de ellas sintomática y que precisa de tratamiento, en las otras no se pautó tratamiento. Los angiomiolipomas han sido manejados de la siguiente manera: nefrectomía simple en 2 pacientes, nefrectomía parcial en 1 paciente, embolización arterial selectiva en 1 paciente y observación en el resto (4). Se presentan 3 casos clínicos representativos y se realiza revisión bibliográfica.

\section{CASOS CLÍNICOS}

\section{Caso clínico 1}

Mujer de 29 años fumadora de medio paquete de cigarrillos diarios. La paciente refiere varios cuadros de neumotórax izquierdos, tratados mediante drenaje torácico y en tratamiento farmacológico con Tamoxifeno y Progesterona. Previamente asintomática, la paciente comienza con cuadro de dolor abdominal difuso, sin otros sintomas, realizándose ecografía abdominal y observando múltiples angiomiolipomas renales bilaterales, entre 6,5 y 7,5 cm en riñón derecho e izquierdo respectivamente. La exploración física era anodina: no presentaba estigmas cutáneos y la exploración neurológica, pulmonar y abdominal fueron normales.

Durante la fase de estudio, la paciente continuó presentando varios episodios de neumotórax, por lo cual se le realizó en abril del 2000, pleurodesis y biopsia pulmonar por videtoracoscopía, llegando al diagnóstico anatomopatológico de linfangioleiomiomatosis pulmonar. Las pruebas de inmunohistoquímica revelaron marcadores positivos de los receptores para estrógenos y para progesterona, siendo más intensos los receptores para estrógenos, todos ellos nucleares. Las pruebas analíticas fueron normales, la función renal conservada y los marcadores inmuno-reumatológicos negativos. La radiografía de tórax demuestra enfermedad pulmonar intersticial con áreas quísticas de predominio en lóbulos superiores (Fig. 1).

En la TAC torácica, se observan múltiples áreas quísticas en ambos campos pulmonares, sin evidencia de adenopatías y en la TAC abdominal vemos tumores renales bilaterales compatibles con angiomiolipomas. En riñón derecho de $6,5 \mathrm{~cm}$ y en polo superior del riñón izquierdo de $7,5 \mathrm{~cm}$. Asimismo, se observa un pequeño quiste en lóbulo hepático derecho. Las pruebas de función pulmonar son normales. Asimismo, la exploración oftalmológica y ginecológica es normal así como la densitometría ósea. Se realizó broncoscopia, sin alteraciones endobronquiales y biopsia transbronquial, sin alteraciones relevantes (Fig. 2). 


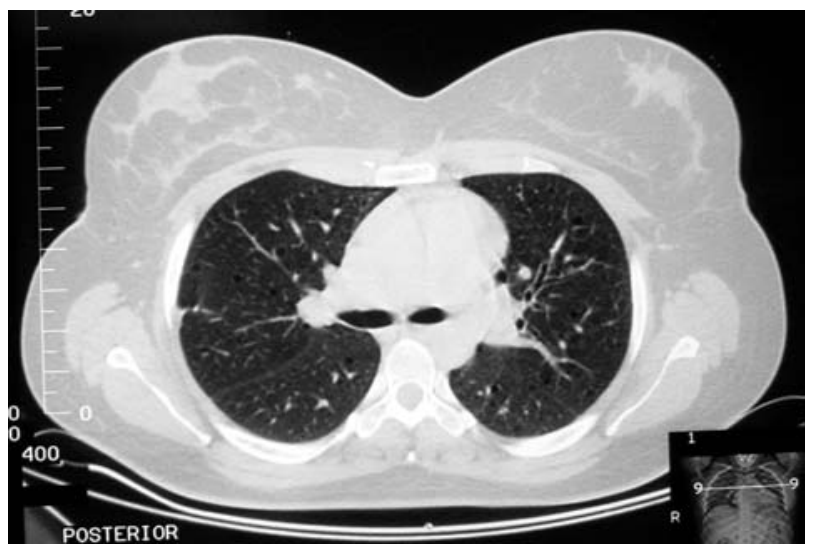

FIGURA 1. TAC Torácico de alta resolución: (2002) Observamos múltiples lesiones quisticas en parénquima pulmonar, afectando difusamente a ambos parénquimas pulmonares, las mayores de $2 \mathrm{~cm}$, en relación con Linfangioleiomiomatosis pulmonar. No se observan adenopatias mediastinicas.

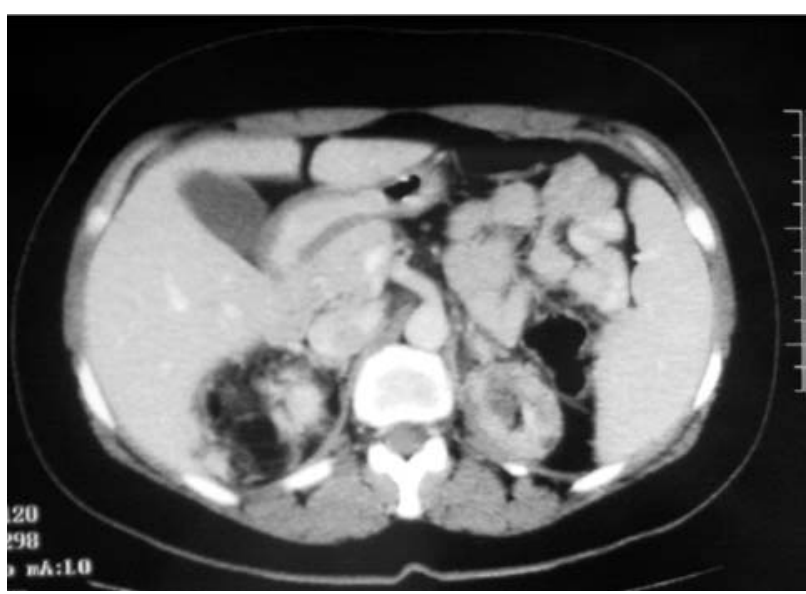

FIGURA 2. TAC Abdominal: (2003) Se observan múltiples masas renales, las mayores en ambos polos superiores de entre 6,5 y $7 \mathrm{~cm}$, con componente sólido y graso, que captan mucho contraste en la fase arterial, compatibles con angiomiolipomas. minución del angiomiolipoma renal izquierdo de 7 a 3 centímetros. Se realiza segunda arteriografía, observando crecimiento del angiomiolipoma renal derecho, de modo que se efectúa embolización consecuente del angiomiolipoma del riñón derecho. En el TAC de control a los 9 meses, se observa disminución de ambos angiomiolipomas, siendo el tamaño de ambos de aproximadamente 3 centímetros. Durante el tratamiento urológico, la paciente continúa presentado cuadros de neumotórax recidivantes, tratados mediante drenaje.

\section{Caso clínico 2}

Mujer de 58 años con antecedentes de síndrome ansioso y epilepsia desde la infancia; Caracterizada por ausencias y convulsiones generalizadas tónico-clónicas, tratada con antiepilépicos. En la RNM cerebral se objetiva lesión nodular temporal izquierda, de 10 x $10 \mathrm{~mm}$ de diámetro.

La paciente consulta el servicio de Digestivo por cuadro de dolor abdominal localizado en hipocondrio derecho, asociado a pérdida de peso de aproximadamente 10 kilos. Los datos analíticos son normales, salvo leve hipercolesterolemia y los marcadores tumorales digestivos son negativos. En la ecografía abdominal se objetiva nódulo de $0,8 \mathrm{~cm}$. en el riñón derecho, isoecogénico con la grasa perirenal. Con este diagnóstico, la paciente es remitida a la consulta externa de Urología. En la TAC abdominal solicitada, se objetiva una masa de $11 \mathrm{~cm}$ de diámetro en el riñón derecho, heterogénea, con áreas grasas, altamente sugestiva de angiomiolipoma, no apre-

Se decide realizar embolización selectiva de ambos angiomiolipomas para evitar hemorragias retroperitoneales espontáneas. En la arteriografía inicial, se observan tres aportes vasculares al angiomiolipoma del riñón derecho y un aporte vascular al angiomiolipoma del riñón izquierdo (Fig. 3).

En una primera sesión, se realiza embolización selectiva del angiomiolipoma renal izquierdo y parcial del derecho. En el control tomográfico a los 3 meses, se observa dis-

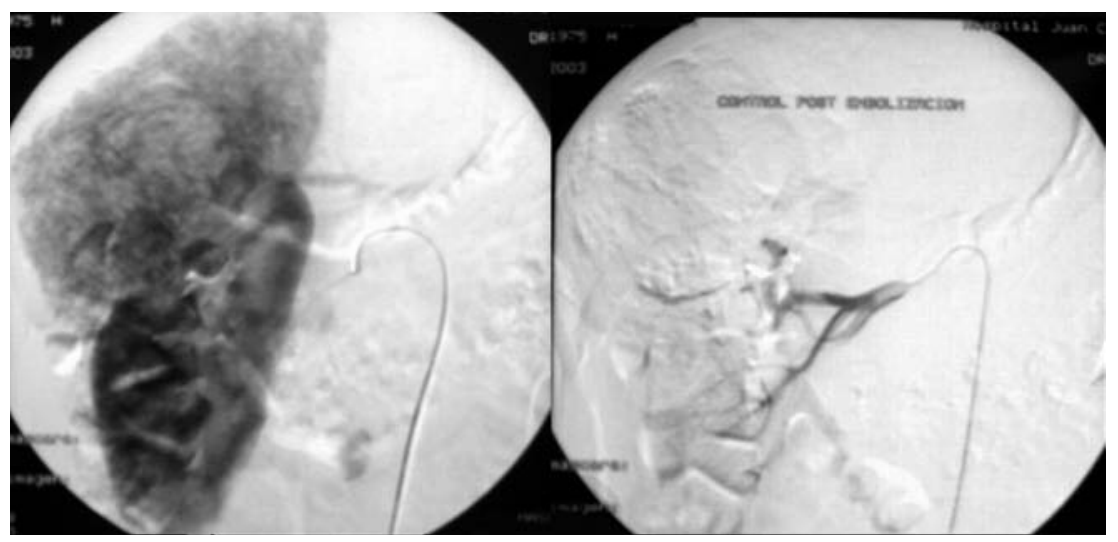

FIGURA 3. Embolización arterial selectiva: a) angiomiolipoma del polo superior en riñón derecho, b) Se evidencia ausencia prácticamente completa de la vascularización tumoral postembolización. 
ciado en la ecografía. Asimismo se observa un nódulo de $1 \mathrm{~cm}$ en polo superior del riñón derecho. En el resto del abdomen se objetivan hamartomas esplénicos de $1 \mathrm{~cm}$, hipodensos. Se realiza en mismo estudio, TAC torácico, apreciándose lesiones quísticas en parénquima pulmonar de menor a $1 \mathrm{~cm}$, que pueden corresponder a una linfangioleiomiomatosis inicial (Fig. 4).

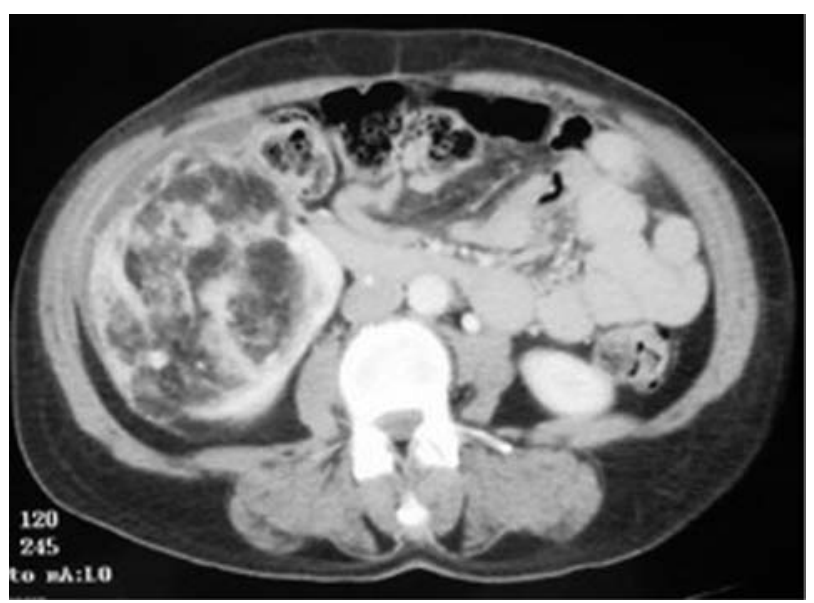

FIGURA 4. TAC Abdominal: Masa de $11 \mathrm{~cm}$. de diámetro en el riñón derecho, heterogénea, con áreas grasas, altamente sugestiva de angiomiolipoma.

Desde el punto de vista respiratorio, se encuentra asintomática. Revisando el historial clínico de la paciente, podemos observar revisiones periódicas en toco-ginecología (G3P2A1) sin hallazgos patológicos. Asimismo aporta revisiones oftalmológicas y neurológicas normales.

Debido al tamaño tumoral y la presencia de síntomas, se realiza nefrectomía simple derecha. La paciente se recupera satisfactoriamente del postoperatorio inmediato y en los controles posteriores, la sintomatología ha desaparecido. La anatomía patológica describe un tumor con las características histológicas e inmunohistoquímicas de angiomiolipoma. En el seguimiento clínico, es valorada por el servicio de Neumología, decidiéndose no instaurar tratamiento hormonal y realizar controles con observación.

\section{Caso clínico 3}

Mujer de 33 años sin antecedentes personales de interés, que consulta por dolor abdominal en hemiabdomen derecho, asociando nauseas y vómitos. En la exploración se detecta masa pal- pable en hipocondrio derecho. En la TAC se observa masa de 11,5 x 9,6 cm de diámetro, sugestiva de angiomiolipoma, en riñón derecho, sin evidencia de sangrado.

Dado el tamaño del tumor, presencia de síntomas, posibilidades de sangrado espontáneo, y sospecha de tumor renal, se realiza nefrectomía derecha, evidenciándose ganglios linfáticos aumentados de tamaño, los cuales se recogen para histología. El resultado anatomopatológico demuestra una tumoración heterogénea sugestiva de angiomiolipoma con áreas epitelioides y con infiltración de 2 ganglios linfáticos con la misma histología.

En el seguimiento clínico la paciente se encuentra asintomática tras 3 años de la cirugía y en la TAC de control al año, no se observan adenopatías retroperitoneales, pero a nivel de los cortes basales pulmonares, se evidencian múltiples lesiones quísticas de distinto tamaño, siendo el parénquima pulmonar circundante de aspecto normal, hallazgo sugestivo de linfangioleiomiomatosis. La paciente refiere disnea de moderados esfuerzo. El servicio de Neumología, decide observación sin instaurar tratamiento.

\section{COMENTARIO}

La afectación renal en el Complejo Esclerosis Tuberosa (TSC), es común y potencialmente seria. En diversas revisiones, la asociación entre TSC y angiomiolipomas, varía entre 47\% y $69 \%$. Cuando se asocian a esta enfermedad, se presentan en personas más jóvenes, bilaterales y multicéntricos. Son más grandes y con mayor tendencia al sangrado. La presencia de quistes renales simples, también es frecuente (30\%-32\%). Los adenocarcinomas se asocian con menos frecuencia $(2,2 \%)^{1-3}$.

Los angiomiolipomas asociados a TSC, presentan mayor tendencia al crecimiento y requieren más intervenciones, que los pacientes con presentación esporádica. De forma que las lesiones con diámetro inicial de al menos $4 \mathrm{~cm}$ tienen más tendencia al crecimiento o intervención que los tumores más pequeños ${ }^{4}$.

La mayoría se detecta de forma incidental, durante un estudio radiológico. Estos tumores tienen predisposición al sangrado, presentándose de manera característica con dolor lumbar, hematuria 
y masa palpable. La clínica puede ser brusca con un sangrado retroperitoneal espontáneo, asociando estado de shock hipovolémico, Esta forma de presentación clínica fue descrita por Wünderlich, al cual este sindrome debe su epónimo y se presenta en el $10 \%$ de los casos.

Los angiomiolipomas pueden ser diagnosticados por su apariencia radiológica. En la ecografia, su apariencia es significativa, por su intensa hiperecogenicidad y sombra acústica posterior, aunque este signo no es patognomónico, ya que entre el $8 \%$ y el $47 \%$ de los pequeños carcinomas renales, son también hiperecogénicos. La ecografia puede usarse como método de seguimiento. El diagnóstico se ajusta mas concretamente con la TAC abdominal, que permite diferenciarlos de los adenocarcinomas renales y sobre todo si produce síntomas ${ }^{5}$.
No existen guías de manejo para el tratamiento de los angiomiolipomas renales, pero las recomendaciones están hechas fundamentalmente en el tamaño tumoral y en la presencia de sínto$\operatorname{mas}^{5-10}$.

Debido a que los tumores mayores de $4 \mathrm{~cm}$, pueden crecer más rápidamente, ser sintomáticos y provocar sangrados, es importante el tamaño para decidir la actitud terapéutica más adecuada y decidir el correcto tratamiento. Los tumores menores de $4 \mathrm{~cm}$ que cursan de manera asintomática, no precisan de tratamiento y pueden ser vigilados anualmente mediante ecografía o TAC. En caso de optar por un tratamiento activo, las opciones terapéuticas son la embolización selectiva arterial o la cirugía conservadora renal (Fig. 5).

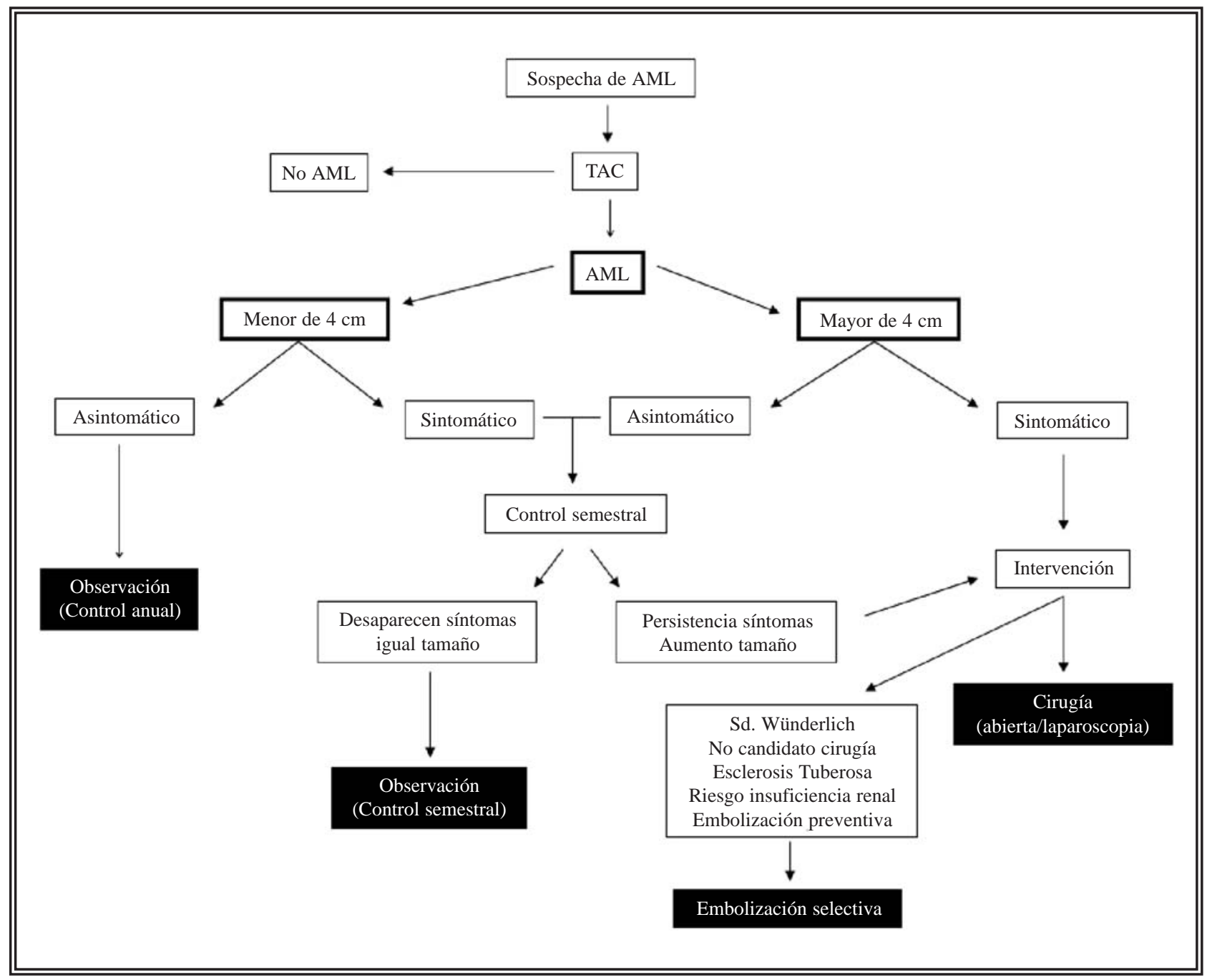

FIGURA 5. Manejo del Angiomiolipoma 
Las principales ventajas de la embolización selectiva, incluyen la conservación del tejido renal circundante, la habilidad de embolizar vasos sangrantes de forma selectiva y suprimir la anestesia general ${ }^{12}$. La embolización arterial selectiva permite controlar los síntomas, de forma efectiva en el 80\%-93\% de los tumores y disminuye la mayoría ${ }^{9}$. Las indicaciones más comunes son el sangrado agudo, los angiomiolipomas sintomáticos en pacientes no candidatos a la cirugía, pacientes con múltiples angiomiolipomas en los cuales la cirugía supondría un riesgo de insuficiencia renal, así como en aquellos que ya tienen escaso tejido funcionante y en pacientes asintomáticos que precisan embolización preventiva $^{10}$. La tasa de complicaciones oscila entre el $0 \%-22 \%^{11}$. La tasa de recidivas es más frecuente en el grupo de pacientes con TSC y están a riesgo de presentar recidivas y nuevos sangrados. En casos de recidiva, las reembolizaciones son efectivas y estos pacientes no son necesariamente subsidiarios de cirugía. Los controles radiológicos deben ser realizados durante toda la vida para identificar a los pacientes que puedan precisar embolizaciones repetidas ${ }^{12}$.

La decisión de intervención quirúrgica en un paciente asintomático, debe ser tomada en conjunto, basada en múltiples factores, entre los que se encuentran el tamaño renal, comorbilidad, presencia de TSC, tejido renal funcionante residual, planificación de embarazo, ocupación laboral, etc.

Los angiomiolipomas representan la manifestación extrapulmonar más frecuente de la linfangioleiomiomatosis. Aparecen hasta en el 60\% de las pacientes jóvenes en edad reproductiva que sufren de linfangioleiomiomatosis, siendo un diagnostico previo en la mayoría de las ocasiones. Mediante el estudio radiológico, se puede caracterizar las lesiones y la TAC de alta resolución (TACAR) es la prueba definitiva para establecer el diagnóstico. El tratamiento de la linfangioleiomiomatosis es generalmente de soporte, dirigido a la obstrucción de la vía aérea, neumotórax de repetición, complicaciones quilosas y sangrados de angiomiolipomas ${ }^{13}$. Según algunos expertos, la terapia debe iniciarse en el diagnóstico, por las características progresivas de la enfermedad. Se debe recomendar la prevención de embarazos o recordar la posibilidad de agravamiento, así como evitar el consumo de preparados estrogénicos. La progesterona y la ooforectomía, en monoterapia o conjuntas, se han demostrado las más eficaces en el manejo ${ }^{14}$. El transplante pulmonar es una opción válida para el tratamiento sustitutivo en la fase final de la enfermedad, con una tasa de supervivencia al primer y segundo año, del 69\% y $58 \%$ respectivamente ${ }^{15}$.

Existe un solapamiento entre los angiomiolipomas renales, la linfangioleiomiomatosis pulmonar y la esclerosis tuberosa; denominándose Complejo Esclerosis Tuberosa. La etiopatogenia es conocida, produciéndose mutaciones en dos genes supresores, el TSC1 (9q34) y TSC2 (16p13). En el TSC, se observa una mutación germinal en los genes TSC1 y TSC2. Cuando se produce una segunda mutación somática, es cuando aparece el desarrollo de la enfermedad. La mayoría de los casos ocurre con mutaciones espontáneas, sin antecedentes parentales. Las mutaciones somáticas del TSC2 y la consiguiente pérdida de heterozigosidad, se observan tanto en las células musculares pulmonares de los pacientes afectados de linfangioleiomiomatosis y en las células renales de los angiomiolipomas ${ }^{16,17}$.

El curso natural de la enfermedad es individual y la intensidad puede ser leve, moderada o severa. Para el diagnóstico de TSC, la Tuberous Sclerosis Alliance ${ }^{18}$ ha desarrollado unos criterios diagnósticos basados en signos clínicos específicos. Para el diagnóstico definitivo de TSC, se precisan un criterio mayor y dos criterios menores. En niños, un criterio mayor y uno menor, establecen un diagnóstico probable y un criterio menor y dos o más mayores, un diagnóstico posible (Tabla 1).

\section{CONCLUSIONES}

La enfermedad de Bourneville es poco frecuente y presenta una rica manifestación urológica de patologías. La más frecuentemente observada es el angiomiolipoma renal. Cuando se asocian a esta enfermedad, los angiomiolipomas suelen ser múltiples, bilaterales, mayor tendentes al crecimiento y requieren más intervenciones. Sus características histológicas son de benignidad, si bien en determinadas ocasiones pueden similar carcinoma renal y precisar de 
Tabla 1

Criterios diagnósticos de Esclerosis Tuberosa (Tuberous Sclerosis Alliance; Julio, 1998)

\begin{tabular}{|c|c|}
\hline \multicolumn{2}{|c|}{ Criterios diagnósticos de Esclerosis Tuberosa } \\
\hline Criterios Mayores & Criterios Menores \\
\hline Angiofibromas faciales o placa frontal & Pits múltiples en esmalte dentario \\
\hline Fibromas ungueales o periungueales no traumáticos & Pólipos rectales hamartomatosos (confirmación histológica) \\
\hline Manchas hipomelanóticas (tres o más) & Quistes óseos (confirmación radiográfica) \\
\hline Placas chagrin & Tractos migratorios en la sustancia blanca cerebral** \\
\hline Hamartomas retinianos nodulares múltiples & Fibromas gingivales \\
\hline Túberes corticales & Hamartoma no renal (confirmación histológica) \\
\hline Nódulos subependimarios & Placa Acrómica en retina \\
\hline Astrocitoma subependimario de células gigantes & Lesiones cutáneas en confeti \\
\hline Rabdomioma cardiaco, único o múltiple & Quistes renales múltiples (confirmación histológica) \\
\hline \multicolumn{2}{|l|}{ Linfangiomiomatosis pulmonar* } \\
\hline \multicolumn{2}{|l|}{ Angiomiolipoma renal* } \\
\hline \multicolumn{2}{|c|}{$\begin{array}{l}\text { * Cuando estos dos criterios estan presentes, se requiere la presencia de otros factores del complejo esclerosis tuberosa (CET) para hacer } \\
\text { un diagnóstico definitivo } \\
\text { ** Cuando existe displasia cortical cerebral y tractos migratorios cerebrales en sustancia blanca de forma conjunta deberán contabilizar- } \\
\text { se como un solo criterio más que como dos criterios separados de CET } \\
\text { Diagnóstico definitivo de CET: Dos criterios mayores o un criterio mayor y dos menores } \\
\text { Diagnótico probable: Un criterio mayor y otro menor } \\
\text { Diagnóstico posible: Un criterio mayor o dos o mas criterios menores }\end{array}$} \\
\hline
\end{tabular}

cirugía. Su manejo es diverso y los tratamientos están orientados a la conservación del parénquima renal circundante $\mathrm{El}$ diagnóstico precoz del angiomiolipoma renal permite prevenir futuras complicaciones mediante la vigilancia, la embolización arterial selectiva o la cirugía conservadora. Es importante el tamaño renal y la presencia de sintomas para decidir la mejor terapia. La embolización selectiva se presenta como la mejor alternativa para la conservación de tejido funcionante, con menor morbilidad.

La linfangioleiomiomatosis pulmonar (LAM) está en ciertas ocasiones asociada a los angiomiolipomas renales y estos pueden llegar a ser sintomáticos y precisar de tratamiento y es por esto que las pacientes con LAM deben ser evaluadas ante la presencia de angiomiolipomas renales. El método diagnóstico ideal es la TAC abdominal. Las pacientes premenopáusicas que han sido diagnosticadas de angiomiolipoma renal, deben ser valoradas ante la posible presencia de linfangioleiomiomatosis pulmonar, mediante la TAC pulmonar de alta resolución (TACAR) y si se confirma, los embarazos deben evitarse o precisar una manipulación hormonal.

\section{REFERENCIAS}

1. Cook JA, Oliver K, Mueller RF, Sampson J. A cross sectional study of renal involvement in tuberous sclerosis. J Med Genet.1996 Jun;33(6):480-484.

2. O'Callaghan FJ, Noakes MJ, Martyn CN, Osborne JP. An epidemiological study of renal pathology in tuberous sclerosis complex. BJU Int. 2004 Oct;94(6):853-857.

3. Bernstein SM, Newell JD Jr, Adamczyk D, Mortenson RL, King TL, Lynch DA. How common are renal angiomiolipomas in patients with pulmonary lymphangiomyomatosis?. Am J Respir Crit Care Med. 1995 Dec;152(6 Pt 1):2138-2143.

4. Steiner MS, Goldman SM, Fishman EK, Marshall FF. The natural history of renal angiomyolipoma. J Urol 1993;150: 1782-1786.

5. Bulto Monteverde JA, Talens A, Navalon P, Garcia Novales JR, Cubells ML, Mendez M. Renal angiomyolipoma. Ultrasonography and computerized tomography findings Arch Esp Urol 1999 Dec;52(10):1043-1050.

6. Dickinson M, Ruckle H, Beaghler M, Hadley HR. Renal angiomyolipoma: optimal treatment based on size and symptoms. Clin Nephrol. 1998 May;49(5):281-286.

7. Heidenreich A, Hegele A, Varga Z, von Knobloch R, Hofmann R. Nephron-sparing surgery for renal angiomyolipoma. Eur Urol. 2002 Mar;41(3):267-273.

8. Rodríguez Vela L, Arino I, Liedana JM, Horndler C, Roncales A, Rioja C, Rioja LA. Bilateral angiomyolipomas of the kidney in Bourneville's tuberous sclerosis. Arch Esp Urol. 1989 Jun;42(5):423-431.

9. Mourikis D, Chatziioannou A, Antoniou A, Kehagias D, Gikas D, Vlahos L. Selective arterial embolization in the management of symptomatic renal angiomyolipomas. Eur J Radiol. 1999 Dec;32(3):153-159. 
10. Nelson CP, Sanda MG. Contemporary diagnosis and management of renal angiomyolipoma. J Urol. 2002 Oct;168(4 Pt 1):1315-1325.

11. Hamlin JA, Smith DC, Taylor FC, McKinney JM, Ruckle HC, Hadley HR. Renal angiomyolipomas: long-term followup of embolization for acute hemorrhage. Can Assoc Radiol J. 1997 Jun;48(3):191-198.

12. Kothary N, Soulen MC, Clark TW, Wein AJ, ShlanskyGoldberg RD, Crino PB et al. Renal angiomyolipoma: longterm results after arterial embolization. $J$ Vasc Interv Radiol. 2005 Jan;16(1):45-50.

13. Johnson SR, Tattersfield AE. Lymphangioleiomyomatosis. Semin Respir Crit Care Med. 2002 Apr;23(2):85-92.

14. Eliasson AH, Phillips YY, Tenholder MF. Treatment of lymphangioleiomyomatosis. A meta-analysis. Chest. 1989 Dec;96(6):1352-1355.

15. Boehler A, Speich R, Russi EW, Weder W. Lung transplantation for lymphangioleiomyomatosis. Engl J Med 1996 Oct; 335(17): 1275-1280.
16. De Pauw RA, Boelaert JR, Haenebalcke CW, Matthys EG, Schurgers MS, De Vriese AS. Renal angiomyolipoma in association with pulmonary lymphangioleiomyomatosis. Am J Kidney Dis. 2003 Apr;41(4):877-883.

17. Yu J, Astrinidis A, Henske EP. Chromosome 16 loss of heterozygosity in tuberous sclerosis and sporadic lymphangiomyomatosis. Am J Respir Crit Care Med. 2001 Oct; 164(8 Pt 1):1537-1540.

18. Roach ES, Gomez MR, Northrup H. Tuberous sclerosis complex consensus conference: revised clinical diagnostic criteria. J Child Neurol. 1998 Dec;13(12):624-628.

Dr. J. Ponce Díaz-Reixa

E-mail: ponce@canalejo.org

(Trabajo recibido el 10 de noviembre de 2005) 